\title{
Current Fluctuations and Electron-Electron Interactions in Coherent Conductors
}

\author{
Artem V. Galaktionov ${ }^{1,3}$, Dmitri S. Golubev ${ }^{2,3}$, and Andrei D. Zaikin ${ }^{1,3}$ \\ ${ }^{1}$ Forschungszentrum Karlsruhe, Institut für Nanotechnologie, 76021, Karlsruhe, Germany \\ ${ }^{2}$ Institut für Theoretische Festkörperphysik, Universität Karlsruhe, 76128 Karlsruhe, Germany \\ ${ }^{3}$ I.E. Tamm Department of Theoretical Physics, P.N. Lebedev Physics Institute, 119991 Moscow, Russia
}

\begin{abstract}
We analyze current fluctuations in mesoscopic coherent conductors in the presence of electronelectron interactions. In a wide range of parameters we obtain explicit universal dependencies of the current noise on temperature, voltage and frequency. We demonstrate that Coulomb interaction decreases the Nyquist noise. In this case the interaction correction to the noise spectrum is governed by the combination $\sum_{n} T_{n}\left(T_{n}-1\right)$, where $T_{n}$ is the transmission of the $n$-th conducting mode. The effect of electron-electron interactions on the shot noise is more complicated. At sufficiently large voltages we recover two different interaction corrections entering with opposite signs. The net result is proportional to $\sum_{n} T_{n}\left(T_{n}-1\right)\left(1-2 T_{n}\right)$, i.e. Coulomb interaction decreases the shot noise at low transmissions and increases it at high transmissions.
\end{abstract}

\section{INTRODUCTION}

Recent advances in nanotechnology enable detailed investigations of a variety of quantum effects in mesoscopic conductors. These investigations are of primary interest because of fundamental importance of such effects as well due to rapidly growing number of their potential applications. A great deal of information is usually obtained from studying electron transport. Additional/complementary information can be extracted from

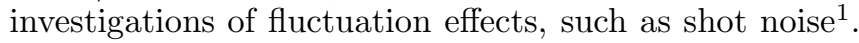
For instance, it was demonstrated23 that the power spectrum of the shot noise in coherent mesoscopic conductors is expressed in terms of the parameter

$$
\beta=\frac{\sum_{n} T_{n}\left(1-T_{n}\right)}{\sum_{n} T_{n}}
$$

Here and below $T_{n}$ stands for the transmission of the $n$ th conducting channel of a coherent conductor. Thus, since transport measurements only allow to determine the combination

$$
\frac{1}{R}=\frac{2 e^{2}}{h} \sum_{n} T_{n}
$$

studies of the shot noise provide additional valuable information about the transmission distribution of conducting modes.

The above results apply to the situations when interaction between electrons can be neglected. In the presence of electron-electron interactions the Landauer conductance (2) and the $I-V$ curve are modified in a nontrivial way. Recently it was shown $\bar{E}$ that the $I-V$ curve of a (comparatively short) coherent conductor with arbitrary transmission distribution $T_{n}$ in the presence of interactions can be expressed in the form

$$
R \frac{d I}{d V}=1-\beta f(V, T),
$$

where $f(V, T)$ is a universal function to be defined below. This result holds in the limit of large conductances
$R \ll R_{q}=h / e^{2}$ or, otherwise, at sufficiently high temperatures/voltages. It demonstrates that the magnitude of the interaction correction is controlled by the same parameter $\beta$ (1) which is already well known in the theory of shot noise. Physically this result can easily be understood since both phenomena are related to discrete nature of the electron charge. Hence, there exists a direct link between shot noise and interaction effects in mesoscopic conductors.

It is obvious that not only the $I-V$ curve (3) but also shot noise as well as higher moments of the current operator should be affected by electron-electron interactions. This paper is devoted to a detailed investigation of current fluctuations in mesoscopic coherent conductors in the presence of electron-electron interactions. Previously various aspects of this problem have been studied for a particular case of tunnel junctions in the Coulomb blockade regime, see, e.g. Refs. 6, 7, 8, 9. The effect of interactions on shot noise in $2 \mathrm{~d}$ diffusive conductors at sufficiently high temperatures was recently addressed in Ref. 10.

Here we will employ a model of a coherent conductor 11.12. Within this model we will demonstrate that interactions lead to two different corrections to the shot noise spectrum. One of these corrections scales with the parameter $\beta$ (1). This correction is always negative, similarly to that found in Eq. (3) for the $I-V$ curve. It describes (partial) suppression of the current noise due to Coulomb blockade. In addition to this correction we shall find another one, which is proportional to the parameter

$$
\gamma=\frac{\sum_{n} T_{n}^{2}\left(1-T_{n}\right)}{\sum_{n} T_{n}}
$$

This second correction is positive, i.e. it leads to relative enhancement of the shot noise. The latter correction turns out to be important only at voltages exceeding both frequency and temperature and is negligible otherwise. Thus, at sufficiently high voltages two interaction corrections - negative and positive - compete, for $\gamma>\beta / 2$ the second one wins and, hence, in this case an overall enhancement of the shot noise by interactions is predicted. 


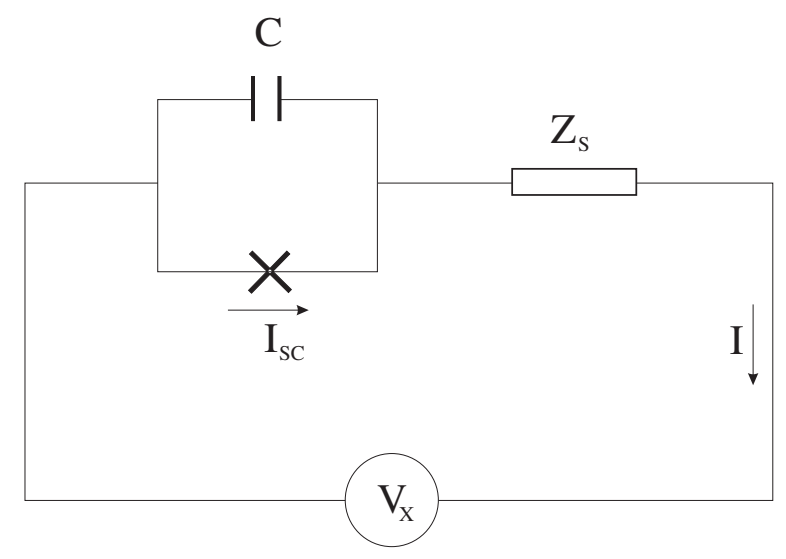

FIG. 1: The circuit under consideration. The scatterer (denoted by a cross) has a capacitance $C$ and is connected to the voltage source $V_{x}$ via an impedance $Z_{S}$.

Our paper is organized as follows. In section 2 we will describe the model and highlight our key results. A detailed derivation of these results is then performed in section 3. Our main conclusions are briefly summarized in section 4. Most of the technical details, such as the derivation of our effective action as well as a few other issues are presented in Appendices A,B and C.

\section{THE MODEL AND KEY RESULTS}

Similarly to Refs. 5, 11, 12 we will consider a coherent scatterer between two big reservoirs. The scatterer is described by an arbitrary distribution of transmissions $T_{n}$ of its conducting modes, and the corresponding Landauer conductance $1 / R$ is defined in Eq. (2). The transmissions $T_{n}$ are assumed to be energy independent. Phase and energy relaxation may take place in these reservoirs but not inside the scatterer, i.e. the scatterer is assumed to be shorter than both dephasing and inelastic lengths. The scatterer region has an effective capacitance $C$. For simplicity we will also assume that the charging energy $E_{C}=e^{2} / 2 C$ does not exceed the typical inverse traversal time. The scatterer is connected to the voltage source $V_{x}$ via linear external impedance $Z_{S}$ (see Fig. 1). Here we restrict ourselves to a simple case $Z_{S}(\omega)=R_{S}$. If necessary, generalization of our calculation to arbitrary $Z_{S}(\omega)$ can be performed in a straightforward manner.

In what follows we will investigate the current noise and evaluate the correlation function

$$
\mathcal{S}\left(t, t^{\prime}\right)=\frac{1}{2}\left\langle\hat{I}(t) \hat{I}\left(t^{\prime}\right)+\hat{I}\left(t^{\prime}\right) \hat{I}(t)\right\rangle-\langle\hat{I}\rangle^{2},
$$

where $\hat{I}$ is the current operator in the circuit of Fig. 1 . This correlator can be expressed in the form

$$
\mathcal{S}\left(t, t^{\prime}\right)=\mathcal{S}^{\mathrm{ni}}\left(t, t^{\prime}\right)+\delta \mathcal{S}\left(t, t^{\prime}\right)
$$

where $\mathcal{S}^{\text {ni }}$ is the noninteracting contribution to the current noise 3 . 1 and $\delta \mathcal{S}$ is the correction due to electron- electron interactions inside the scatterer. This correction will be evaluated in the most interesting "metallic" limit

$$
g_{0}=g+g_{S} \gg 1 .
$$

Here we introduced dimensionless conductances of the scatterer and the shunt, respectively $g=R_{q} / R$ and $g_{S}=$ $R_{q} / R_{S}$. Eq. (7) implies that at least one of these two dimensionless conductances is required to be much larger than unity.

Quite obviously, the latter correlator (5) should depend on both $R$ and $R_{S}$. We also introduce another correlator $\tilde{\mathcal{S}}\left(t, t^{\prime}\right)$ defined by the same Eq. (5) in which one should substitute the current operator across the scatterer $\hat{I} \rightarrow \hat{I}_{\mathrm{sc}}$. The two correlators $\mathcal{S}\left(t, t^{\prime}\right)$ and $\tilde{\mathcal{S}}\left(t, t^{\prime}\right)$ are not independent. With the aid of the current conservation condition and performing the Fourier transformation with respect to $t-t^{\prime}$, one easily finds the relation

$$
\begin{aligned}
\tilde{\mathcal{S}}_{\omega}= & \frac{R_{S}^{2}}{R_{0}^{2}}\left(1+\omega^{2} R_{0}^{2} C^{2}\right) \mathcal{S}_{\omega} \\
& -\frac{R_{S}}{R^{2}}\left(1+\omega^{2} R^{2} C^{2}\right) \omega \operatorname{coth} \frac{\omega}{2 T},
\end{aligned}
$$

where $R_{0}=R R_{S} /\left(R+R_{S}\right)$. The second term is due the noise produced by the external resistor $R_{S}$ which has to be subtracted in order to arrive at $\tilde{\mathcal{S}}_{\omega}$.

In general also the correlator $\tilde{\mathcal{S}}_{\omega}$ depends on both $R$ and $R_{S}$. However, in the limit $R_{S} \gg R$ the dependence on the shunt resistance is weak and can be neglected. In this case the interaction correction to the current noise spectrum $\delta \tilde{\mathcal{S}}_{\omega}$ depends only on the properties of the scatterer. Below we will present our key results for $\delta \tilde{\mathcal{S}}_{\omega}$ only in this limit. More general expressions can be found in Sec. 3.

Let us define the average voltage across the scatterer $V=V_{x} R /\left(R+R_{S}\right)$ and consider first the limit of relatively small voltages. At sufficiently large temperatures and/or frequencies we find

$$
\begin{aligned}
\delta \tilde{\mathcal{S}}_{\omega}=-\frac{2 \beta E_{C}}{3 R}, & \text { if } T \gg g E_{C},|e V|,|\omega|, \\
\delta \tilde{\mathcal{S}}_{\omega}=-\frac{\beta E_{C}}{R}, & \text { if }|\omega| \gg T, g E_{C},|e V| .
\end{aligned}
$$

At lower temperatures and frequencies we obtain

$$
\begin{gathered}
\delta \tilde{\mathcal{S}}_{\omega}=-\frac{4 \beta T}{R_{q}} \ln \frac{g E_{C}}{T}, \quad \text { if }|\omega|,|e V| \ll T \ll g E_{C}, \\
\delta \tilde{\mathcal{S}}_{\omega}=-\frac{2 \beta|\omega|}{R_{q}} \ln \frac{g E_{C}}{|\omega|}, \quad \text { if } T,|e V| \ll|\omega| \ll g E_{C} .
\end{gathered}
$$

These results apply as long as either temperature or frequency exceeds an exponentially small parameter $g E_{C} \exp \left(\bar{n}^{g / 2}\right)$. For even smaller frequencies and temperaturest 4 we get

$$
\delta \tilde{\mathcal{S}}_{\omega}=-\frac{\beta \omega}{R} \operatorname{coth} \frac{\omega}{2 T} .
$$


Note that the above expressions could also be anticipated from the fluctuation-dissipation theorem (FDT) combined with the results]. Indeed, in the limit of low voltages the current noise is described by the standard Nyquist formula. Hence, in order to satisfy FDT one should simply substitute the effective conductance (3) into this formula. In this way one gets the interaction correction $\delta \tilde{S}_{\omega}$ proportional to $\beta f$. For instance, in the low frequency limit one finds $f(0, T) \simeq E_{C} / 3 T$ for $T \gg g E_{C}, f(0, T) \simeq(2 / g) \ln \left(g E_{C} / T\right)$ for $\exp (-g / 2) \ll$ $T / g E_{C} \ll 1$ and $f(0, T) \simeq 1$ for $T<g E_{C} \exp (-g / 2)$. Combining these expressions with FDT one immediately reproduces Eqs. (9), (11) and (13).

It is worth stressing that here we evaluate the currentcurrent correlation functions directly and do not use the results 5 together with FDT. However, it is satisfactory to observe that FDT is explicitly maintained in our calculation and the results derived here are fully consistent with those of Ref. 5 .

Now let us turn to the case of relatively large voltages $V$ where the shot noise becomes important. As it was already announced, in this case the correction to the noise power spectrum is proportional to the parameter

$$
\beta-2 \gamma=\frac{\sum_{n} T_{n}\left(1-T_{n}\right)\left(1-2 T_{n}\right)}{\sum_{n} T_{n}} .
$$

In particular we obtain

$$
\begin{array}{r}
\delta \tilde{\mathcal{S}}_{\omega}=-\frac{2(\beta-2 \gamma)|e V|}{R_{q}} \ln \frac{g E_{C}}{|e V|}, \\
\text { if } T,|\omega| \ll|e V| \ll g E_{C}, \\
\delta \tilde{S}_{\omega}=-\frac{(\beta-2 \gamma) E_{C}}{R}, \quad \text { if }|e V| \gg T, g E_{C},|\omega| .
\end{array}
$$

We note that this correction can be either negative or positive depending on the relation between the parameters $\beta$ and $\gamma$. Thus, in contrast to the limit of low voltages (Nyquist noise), one cannot conclude that shot noise is always reduced by interactions. This reduction occurs only for conductors with relatively low transmissions $\beta>2 \gamma$, while for systems with higher transmissions the net effect of the electron-electron interaction enhances the shot noise. In the important case of diffusive conductors one has $\beta=1 / 3, \gamma=2 / 15$ and, hence,

$$
\beta-2 \gamma=\frac{1}{15}
$$

In this case the shot noise is reduced by interactions.

The above results have a transparent physical interpretation. At low voltages the power spectrum of the Nyquist noise is proportional to the system conductance $\propto \sum_{n} T_{n}$. Since in the presence of interactions the conductance acquires a correction proportional to $\beta$, the interaction correction to the Nyquist noise should scale with the same parameter (11). On the other hand, shot noise is determined by the combination $\sum_{n}\left(T_{n}-T_{n}^{2}\right)$. Accordingly, the interaction correction to the shot noise power should consist of two contributions. One of them comes from $\sum_{n} T_{n}$ and is again proportional to $\beta$. Another contribution originates from the interaction correction to $\sum_{n} T_{n}^{2}$ which turns out to scale as $2 \gamma$. Since these two corrections enter with the opposite signs we immediately arrive at the combination (14).

We also point out that the third cumulant of the current operator for noninteracting electrons is known 13 to be proportional to the parameters (11) and (14) respectively at low and high voltages. Following the same arguments as above we can conjecture that the interaction correction to the third cumulant should scale as $\beta-2 \gamma$ at low voltages, while in the limit of large voltages one can expect that this correction is governed by the combination $\beta-6 \gamma+6 \delta$, where

$$
\delta=\frac{\sum_{n} T_{n}^{3}\left(1-T_{n}\right)}{\sum_{n} T_{n}} .
$$

This conjecture can also be generalized to higher cumulants of the current operator.

We would like to emphasize that - although the above conjecture seems intuitively appealing - it should still be verified by means of a rigorous calculation which is beyond the scope of the present paper. In the next section we will concentrate on the current noise and will provide a detailed derivation of the results presented above.

\section{EFFECTIVE ACTION AND CURRENT NOISE}

Similarly to Ref. 目we will use the effective action technique in order to evaluate the current-current correlator for the system depicted in Fig. 1. It is convenient to introduce the quantum phase variable $\varphi$ which is proportional to the integral of the fluctuating voltage (see Appendix A). We will proceed within the Keldysh formalism and introduce two phase variables $\varphi_{1,2}$ related to the two branches of the Keldysh contour. Defining $\varphi^{+}=\left(\varphi_{1}+\varphi_{2}\right) / 2$ and $\varphi^{-}=\varphi_{1}-\varphi_{2}$ one can denote the overall phase jumps across the scatterer as $\varphi^{+}+e V t$ and $\varphi^{-}$. Correspondingly, the phase jumps across the Ohmic shunt are $\left(e V_{x}-e V\right) t-\varphi^{+}$and $-\varphi^{-}$. The symmetric current-current correlation function (5) can be expressed as follows

$$
\begin{array}{r}
\frac{1}{2}\left\langle\hat{I}(t) \hat{I}\left(t^{\prime}\right)+\hat{I}\left(t^{\prime}\right) \hat{I}(t)\right\rangle=-e^{2} \int \mathcal{D} \varphi^{ \pm} \\
{\left[\frac{\delta^{2}}{\delta \varphi_{S}^{-}(t) \delta \varphi_{S}^{-}\left(t^{\prime}\right)}+\frac{\delta^{2}}{4 \delta \varphi_{S}^{+}(t) \delta \varphi_{S}^{+}\left(t^{\prime}\right)}\right] e^{i S_{\mathrm{tot}}\left[\varphi^{ \pm}\right]},}
\end{array}
$$

see Appendix A for further discussion. By $\varphi_{S}^{ \pm}$we denote the phase jumps over the ohmic shunt. The variational derivatives in Eq.(18) act on the shunt part of action. Here $S_{\text {tot }}\left[\varphi^{ \pm}\right]$is the total action of our system

$$
S_{\mathrm{tot}}\left[\varphi^{ \pm}\right]=S\left[\varphi^{ \pm}\right]+S_{S}\left[\varphi^{ \pm}\right]
$$


where the term

$$
\begin{gathered}
i S_{S}\left[\varphi^{ \pm}\right]=\frac{i}{e^{2} R_{S}} \int_{0}^{\infty} d t \varphi^{-}(t)\left(\frac{e V_{x} R_{S}}{R+R_{S}}-\dot{\varphi}^{+}(t)\right) \\
-\frac{1}{2 e^{2} R_{S}} \int_{0}^{\infty} d t_{1} \int_{0}^{\infty} d t_{2} \alpha\left(t_{1}-t_{2}\right) \varphi^{-}\left(t_{1}\right) \varphi^{-}\left(t_{2}\right)
\end{gathered}
$$

comes from the shunt, $S\left[\varphi^{ \pm}\right]$is the scatterer action and

$$
\alpha\left(t-t^{\prime}\right)=-\frac{1}{\pi}\left(\frac{\pi T}{\sinh \left[\pi T\left(t-t^{\prime}\right)\right]}\right)^{2} .
$$

A detailed derivation of the action $S\left[\varphi^{ \pm}\right]$is carried out in Appendices A, B and C. The main idea of this derivation is to expand $S\left[\varphi^{ \pm}\right]$in powers of $\varphi^{-}$keeping the full nonlinearity of the corresponding terms in $\varphi^{+}$. This procedure is just the quasiclassical approximation for the phase variable. It is parametrically justified under the condition (7). In Ref. 5 the action $S\left[\varphi^{ \pm}\right]$was evaluated up to the second order in $\varphi^{-}$. This is sufficient to derive the current-voltage characteristics of the scatterer. However, in order to describe the current noise it is necessary to expand the action $S\left[\varphi^{ \pm}\right]$further and to retain all terms up to the third order in $\varphi^{-}$

$$
S\left[\varphi^{ \pm}\right]=S^{(1)}+S^{(2)}+S^{(3)} .
$$

This expansion is analyzed in Appendix C. We will now use these results and explicitly evaluate the currentcurrent correlator (5).

\section{A. Contribution of first and second order terms}

Let us first restrict our attention to the contribution of the first and second order terms in (22). They read

$$
\begin{array}{r}
i S^{(1)}\left[\varphi^{ \pm}\right]+i S^{(2)}\left[\varphi^{ \pm}\right]=-\frac{i}{e^{2}} \int_{0}^{\infty} d t \varphi^{-}(t)\left[C \ddot{\varphi}^{+}(t)+\frac{1}{R}\left(\dot{\varphi}^{+}(t)+e V\right)\right] \\
-\frac{1}{2 e^{2} R} \int_{0}^{\infty} d t_{1} \int_{0}^{\infty} d t_{2} \alpha\left(t_{1}-t_{2}\right) \varphi^{-}\left(t_{1}\right) \varphi^{-}\left(t_{2}\right)\left\{1-\beta+\beta \cos \left[e V\left(t_{1}-t_{2}\right)+\varphi^{+}\left(t_{1}\right)-\varphi^{+}\left(t_{2}\right)\right]\right\} .
\end{array}
$$

Employing Eq. (18) we obtain

$$
\frac{1}{2}\left\langle\hat{I}(t) \hat{I}\left(t^{\prime}\right)+\hat{I}\left(t^{\prime}\right) \hat{I}(t)\right\rangle=\frac{\alpha\left(t-t^{\prime}\right)}{R_{S}}+e^{2}\left\langle K(t) K\left(t^{\prime}\right)\right\rangle
$$

where we defined

$$
K(t)=\frac{1}{e^{2} R_{S}}\left[e V_{x}-e V-\dot{\varphi}^{+}+i \int_{0}^{\infty} d \tilde{t} \alpha(t-\tilde{t}) \varphi^{-}(\tilde{t})\right] .
$$

Note that here we neglected terms which originate from the second variational derivative with respect to $\varphi^{+}$in (18) since these terms are smaller in the parameter $1 / g_{0}^{2}$ than those kept. Angular brackets in Eq. (23) imply averaging with the path integral

$$
\langle\ldots\rangle=\int \mathcal{D} \varphi^{ \pm}(\ldots) \exp \left(i S^{(1)}\left[\varphi^{ \pm}\right]+i S^{(2)}\left[\varphi^{ \pm}\right]+i S_{S}\left[\varphi^{ \pm}\right]\right) .
$$

Rewriting the correlator $\langle K K\rangle$ as

$$
\left\langle K(t) K\left(t^{\prime}\right)\right\rangle=-\lim _{\eta \rightarrow 0} \int \mathcal{D} \varphi^{ \pm} \frac{\delta^{2}}{\delta \eta(t) \delta \eta\left(t^{\prime}\right)} \exp \left\{i \sum_{i=1,2} S^{(i)}\left[\varphi^{ \pm}\right]+i S_{S}\left[\varphi^{ \pm}\right]+i \int_{0}^{\infty} d \tilde{t} \eta(\tilde{t}) K(\tilde{t})\right\}
$$

and performing a shift of $\varphi_{\omega}^{-} \rightarrow \varphi_{\omega}^{-}+\eta_{\omega} R_{0} / R_{s}\left(1-i \omega R_{0} C\right)$, we obtain the expression for the Fourier transformed noise spectrum (5)

$$
\begin{aligned}
\mathcal{S}_{\omega}= & \omega \operatorname{coth} \frac{\omega}{2 T}\left\{\operatorname{Re} \frac{1}{Z(\omega)}-\frac{\beta}{R \kappa \Omega}\right\}+\frac{\beta\left[\alpha\left(t-t^{\prime}\right)\left\langle\cos \left(e V\left(t-t^{\prime}\right)+\varphi^{+}(t)-\varphi^{+}\left(t^{\prime}\right)\right)\right\rangle\right]_{\omega}}{R \kappa \Omega}+ \\
& +\frac{\beta^{2}}{e^{2} R^{2} \kappa \Omega}\left[\langle H(t)\rangle^{2}-\left\langle H(t) H\left(t^{\prime}\right)\right\rangle\right]_{\omega},
\end{aligned}
$$

where

$$
H(t)=\int_{0}^{\infty} d \tilde{t} \alpha(t-\tilde{t}) \varphi^{-}(\tilde{t})\left[1-\cos \left(e V(t-\tilde{t})+\varphi^{+}(t)-\varphi^{+}(\tilde{t})\right)\right]
$$


In Eq. (26) we also introduced the following notations

$$
Z(\omega)=R_{S}+\frac{1}{R^{-1}-i \omega C}, \quad \Omega=1+\omega^{2} R_{0}^{2} C^{2}, \quad \kappa=\frac{\left(R+R_{S}\right)^{2}}{R^{2}} .
$$

Making use of the relation ( 8 , we arrive at the correlator $\tilde{S}_{\omega}$,

$$
\tilde{\mathcal{S}}_{\omega}=\frac{1}{R}\left((1-\beta) \omega \operatorname{coth} \frac{\omega}{2 T}+\beta\left[\alpha\left(t-t^{\prime}\right)\left\langle\cos \left(e V\left(t-t^{\prime}\right)+\varphi^{+}(t)-\varphi^{+}\left(t^{\prime}\right)\right)\right\rangle\right]_{\omega}\right)+\ldots
$$

where ... stands for the terms containing $\left[\langle H(t)\rangle^{2}-\left\langle H(t) H\left(t^{\prime}\right)\right\rangle\right]_{\omega}$ in Eq. 26.

Within our approach interaction effects are described by the terms containing the fluctuating variable $\varphi^{+}$. If one formally sets this variable equal to zero, from (26) one immediately recovers the noninteracting result 2 , 1 , 6

$$
\tilde{\mathcal{S}}_{\omega}^{\text {ni }}=(1-\beta) \frac{\omega}{R} \operatorname{coth} \frac{\omega}{2 T}+\frac{\beta}{2} \sum_{ \pm}(\omega \pm e V) \operatorname{coth} \frac{\omega \pm e V}{2 T} .
$$

Taking the phase fluctuations into account we arrive at the expression for the interaction correction to (30). However, the corresponding expression turns out to be incomplete in two respects. First, one of the terms does not satisfy FDT. Second, the correction to (30) obtained in this way scales with the parameter $\beta$ in both limits of small and large voltages. While in the former limit (Nyquist noise) this result is understandable and consistent with Ref. 5, at large voltages (shot noise) one also expects an extra contribution. Its existence can be anticipated because the shot noise is governed by the combination $\sum_{n} T_{n}\left(1-T_{n}\right)$ and not simply by $\sum_{n} T_{n}$ as the Nyquist noise, see also our discussion in Sec. 2.

Both these problems are remedied by taking into account the third order in $\varphi^{-}$contribution to the effective action. This will be demonstrated in the next subsection.

\section{B. Corrections due to third order terms}

Following the analysis in Appendix $\mathrm{C}$ we identify two different contributions to the third order term

$$
S^{(3)}=S_{\beta}^{(3)}+S_{\gamma}^{(3)}
$$

The first contribution has the form

$$
i S_{\beta}^{(3)}\left[\varphi^{ \pm}\right]=\frac{i \beta}{6 e^{2} R} \int_{0}^{\infty} d \tau\left(\varphi^{-}(\tau)\right)^{3} \dot{\varphi}^{+}(\tau)
$$

Taking this term into account and repeating the above analysis we arrive at an extra contribution to the current noise in the form $-e^{2} \beta \delta\left(t-t^{\prime}\right) / 2 R C$, see also Appendix C. Adding this contribution to Eq. (26) and subtracting the noninteracting result (30) we arrive at the interaction correction

$$
\delta S_{\omega}^{(\beta)}=\frac{\beta}{R \kappa \Omega}\left[\alpha\left(t-t^{\prime}\right) \cos \left(e V\left(t-t^{\prime}\right)\right)\left(e^{-F\left(t-t^{\prime}\right)}-1\right)-e^{2} \delta\left(t-t^{\prime}\right) / 2 C\right]_{\omega} .
$$

The function $F(t)$ results from averaging over the phase fluctuations

$$
\left\langle\cos \left[e V\left(t-t^{\prime}\right)+\varphi^{+}(t)-\varphi^{+}\left(t^{\prime}\right)\right]\right\rangle=\cos \left[e V\left(t-t^{\prime}\right)\right] e^{-F\left(t-t^{\prime}\right)} .
$$

This function has the form

$$
F(t)=e^{2} R_{0}^{2} \int_{-\infty}^{\infty} \frac{d \omega}{2 \pi} \frac{1-\cos \omega t}{\omega^{2} \Omega}\left\{\left(\frac{1}{R_{0}}-\frac{\beta}{R}\right) \omega \operatorname{coth} \frac{\omega}{2 T}+\frac{\beta}{2 R} \sum_{ \pm}(\omega \pm e V) \operatorname{coth} \frac{\omega \pm e V}{2 T}\right\} .
$$

We also note that in Eq. (32) we omitted the last term of Eq. (26) which contains averages of the function $H$ (27). Our analysis demonstrates that these terms are small in all the regimes considered below. 
What remains is to evaluate the correction to the shot noise from the second contribution to $S^{(3)}$. The derivation of this contribution is presented in Appendix C. Here we only quote the result:

$$
\begin{array}{r}
i S_{\gamma}^{(3)}\left[\varphi^{ \pm}\right]=\frac{\pi i \gamma T^{3}}{6 e^{2} R} \int_{0}^{\infty} d y_{1} \int_{0}^{\infty} d y_{2} \int_{0}^{\infty} d y_{3} \frac{\varphi^{-}\left(y_{1}\right) \varphi^{-}\left(y_{2}\right) \varphi^{-}\left(y_{3}\right)}{\sinh \left[\pi T y_{21}\right] \sinh \left[\pi T y_{32}\right] \sinh \left[\pi T y_{13}\right]} \times \\
\left\{\sin \left(e V y_{21}+\varphi^{+}\left(y_{2}\right)-\varphi^{+}\left(y_{1}\right)\right)+\sin \left(e V y_{32}+\varphi^{+}\left(y_{3}\right)-\varphi^{+}\left(y_{2}\right)\right)+\sin \left(e V y_{13}+\varphi^{+}\left(y_{1}\right)-\varphi^{+}\left(y_{3}\right)\right)\right\}
\end{array}
$$

where $y_{i j}=y_{i}-y_{j}$ and the parameter $\gamma$ is defined in Eq. (四).

At the first glance this contribution to the effective action could be considered unimportant. This is indeed the case in several limits. For instance, at sufficiently small transmissions $\beta \gg \gamma$ the term (35) can obviously be neglected. In the limit of low voltages one can, making use of the condition (7), expand $S_{\gamma}^{(3)}$ in small phase fluctuations $\varphi^{ \pm}$. Then one gets $S_{\gamma}^{(3)}$ proportional to the combination $\left(\varphi^{+}\right)^{3}\left(\varphi^{-}\right)^{3}$ which can be dropped as compared to other terms provided $g_{0} \gg 1$. However, in the limit of large voltages the term (35) gains importance and - as we shall see provides significant contribution to $\delta \mathcal{S}_{\omega}$.

Proceeding along the lines with the above analysis we find

$$
\begin{aligned}
\delta \mathcal{S}_{\omega}^{(\gamma)}= & \frac{2 \pi^{2} \gamma T^{3}}{g_{0} R \kappa \Omega} \times \\
& \int_{0}^{\infty} d t \int_{0}^{\infty} d x \frac{\left(1-e^{-x / R_{0} C}\right)(\cos [e V t]-\cos [e V x]) \cos \omega t}{\sinh [\pi T x] \sinh [\pi T t]}\left(\frac{1}{\sinh [\pi T(x-t)]}-\frac{1}{\sinh [\pi T(x+t)]}\right) .
\end{aligned}
$$

This expression will be analyzed below in Sec. 3F.

\section{Relation to FDT}

Before we proceed with the analysis of the above results let us establish some useful general expressions and illustrate the relation between our approach and FDT. Throughout this subsection we will only consider the limit of small voltages $\mathrm{eV} \ll 1 / R_{0} C$ and neglect the dependence of the function $F(t)$ on $V$. In the spirit of the $P(E)$-theory 15 let us define the function

$$
\begin{array}{r}
P(E)=\int_{-\infty}^{\infty} d t e^{i E t} e^{-\Phi(t)}, \\
\Phi(t)=\left.F(t)\right|_{V=0}+\frac{i e^{2} R_{0}}{2} \operatorname{sign}[t]\left(1-e^{-|t| / R_{0} C}\right) .
\end{array}
$$

This function obeys the "detailed balance" symmetry $P(-E)=e^{-E / T} P(E)$ which follows from the property $\Phi(t-(i / T))=\Phi(-t)$. Let us also introduce the function

$$
\begin{array}{r}
\mathcal{N}_{\omega}=\frac{1}{4 \pi R_{0}} \times \\
\sum_{ \pm} \int_{-\infty}^{\infty} d E \frac{E\left(1+e^{-(\omega \pm e V) / T}\right)}{1-e^{-E / T}} P(\omega \pm e V-E),
\end{array}
$$

and rewrite it in the form

$$
\begin{array}{r}
\mathcal{N}_{\omega}=\frac{1}{R_{0}} \int_{-\infty}^{\infty} d t e^{i \omega t}\left\{-\frac{e^{2}}{2 C} \delta(t)+\right. \\
\left.\alpha(t) e^{-F(t)} \cos (e V t) \cos \left[\frac{\pi}{g_{0}}\left(1-e^{-t / R_{0} C}\right)\right]\right\} .
\end{array}
$$

We observe that, since in the interesting for us limit $g_{0} \gg$ 1 the argument of $\cos \left[\frac{\pi}{g_{0}}\left(1-e^{-t / R_{0} C}\right)\right]$ is small, with the accuracy $\sim 1 / g_{0}^{2}$ one can use the function (39) in order to analyze the result (32).

Proceeding further let us rewrite Eq. (38) as

$$
\mathcal{N}(\omega)=\frac{1}{2} \sum_{ \pm} \operatorname{coth} \frac{\omega \pm e V}{2 T} \mathcal{I}(\omega \pm e V)
$$

where

$$
\mathcal{I}(\omega)=\frac{1-e^{-\omega / T}}{2 \pi R_{0}} \int_{-\infty}^{\infty} \frac{d E E}{1-e^{-E / T}} P(\omega-E) .
$$

After a simple algebra from Eq. (41) we obtain $\mathcal{I}(\omega)=$ $\frac{\omega}{R_{0}}+\delta \mathcal{I}(\omega)$ and

$$
\begin{array}{r}
\delta \mathcal{I}(\omega)=\frac{2}{R_{0}} \times \\
\int_{0}^{\infty} d t \sin (\omega t) e^{-F(t)} \alpha(t) \sin \left[\frac{\pi}{g_{0}}\left(1-e^{-t / R_{0} C}\right)\right] .
\end{array}
$$

Comparing the above expressions with Eq. (32) we arrive at the following correction to the current noise

$$
\delta \mathcal{S}_{\omega}=\frac{\beta R_{0}}{2 R \kappa \Omega} \sum_{ \pm} \operatorname{coth} \frac{\omega \pm e V}{2 T} \delta \mathcal{I}(\omega \pm e V) .
$$

In order to illustrate the relation between our results and FDT we notice that in the relevant limit $g_{0} \gg 1$ the quantity $\delta \mathcal{I}(\mathrm{eV})(42)$ is defined by exactly the same time integral as the interaction correction to the $I-V$ curve, cf. Eq. (27) of Ref. 5. In particular, in the limit of zero frequency and voltage one finds

$$
\delta \tilde{\mathcal{S}}_{\omega=0}=2 T e^{2} \beta \int_{0}^{\infty} t \alpha(t) e^{-F(t)}\left(1-e^{-t / R_{0} C}\right) d t .
$$


In accordance with FDT the combination in the right hand side is just the interaction correction to the zerobias conductance of a coherent scatteren 5 multiplied by $2 T$.

We will now derive the interaction correction to the current noise in several important limits.

\section{High temperatures}

In the limit $T \gg 1 / R_{0} C$ it is sufficient to evaluate the function $F(t)$ only at short times $t \lesssim 1 / T$. In this limit from Eq. (34) we get

$$
F(t)=\frac{e^{2} t^{2}}{2 C}\left[\left(1-\frac{\beta R_{0}}{R}\right) T+\frac{\beta R_{0}}{2 R} e V \operatorname{coth} \frac{e V}{2 T}\right] .
$$

Expanding $e^{-F(t)}$ in Eq. (32) to the first order in $F$ we obtain

$$
\begin{array}{r}
\delta \mathcal{S}_{\omega}=\frac{e^{2} \beta}{2 R C \kappa \Omega}\{-1+ \\
\left.\left[1+\frac{\beta R_{0}}{R}\left(\frac{e V}{2 T} \operatorname{coth} \frac{e V}{2 T}-1\right)\right] \sum_{ \pm} f\left(\frac{\omega \pm e V}{2 T}\right)\right\}
\end{array}
$$

where function $f(x)$ reads

$$
f(x)=\frac{x \cosh x}{2 \sinh ^{3} x}-\frac{1}{2 \sinh ^{2} x} .
$$

In the limit of small frequencies and voltages we then find

$$
\delta \mathcal{S}_{\omega}=-\frac{e^{2} \beta}{3 R C \kappa}
$$

At high frequencies $\omega \gg T, e V$ or large voltages $e V \gg$ $T, \omega$ we get

$$
\delta \mathcal{S}_{\omega}=-\frac{e^{2} \beta}{2 R C \kappa \Omega} .
$$

Both results (48) and (49) describe partial suppression of the current noise by Coulomb interaction. As we have already discussed, Eq. (48) is consistent with the results combined with FDT, whereas Eq. (49) just corresponds to the Coulomb offset $\Delta V=-e \beta / 2 C$ on the $I-V$ curve of a coherent scatterer at large voltages. For the sake of completeness we also note that in a specific limit $|\omega \pm e V| \ll T$ Eq. (46) yields positive correction to the current noise

$$
\delta \mathcal{S}_{\omega}=\frac{e^{2} \beta^{2} R_{0}|e V|}{24 C T R^{2} \kappa \Omega}
$$

However, the magnitude of this correction is small in the parameter $\sim e^{2} R_{0} /(R C T) \ll 1 / g_{0}$.

\section{E. Low temperatures}

Now let us consider the limit of low temperatures $T \ll$ $1 / C R_{0}$. At low voltages $e V \ll 1 / R_{0} C$ and times much longer than $1 / R_{0} C$ the function $F(t)$ reads

$$
F(t) \simeq \frac{2}{g_{0}} \ln \left(\frac{\sinh [\pi T t]}{\sinh \left[\pi T R_{0} C\right]}\right) .
$$

Combining this expression with Eq. (32), in the limit of small $\omega, e V<T$ we obtain

$$
\mathcal{S}_{\omega}=2 T\left[\frac{1}{R+R_{S}}-\frac{\beta}{R \kappa}\left(1-\left(T R_{0} C\right)^{\frac{2}{g_{0}}}\right)\right] .
$$

For $T \gg g_{0} E_{C} \exp \left(-g_{0} / 2\right)$ the result (52) can be expanded in $2 / g_{0}$. In this limit for the interaction correction we get

$$
\delta \mathcal{S}_{\omega}=-\frac{4 T \beta}{g_{0} R \kappa} \ln \frac{1}{R_{0} C T} .
$$

In the opposite limit of very low $T<g_{0} E_{C} \exp \left(-g_{0} / 2\right)$ (but still $T \gg \omega, e V$ ) the last term in (52) can be neglected and the interaction correction becomes

$$
\delta \mathcal{S}_{\omega}=-\frac{2 T \beta}{R \kappa}
$$

In the limit $T \ll \omega, e V \ll 1 / R_{0} C$ we can set $T=0$ in Eq. (51). Then we obtain

$$
\begin{array}{r}
\mathcal{S}_{\omega}=|\omega|\left(\frac{1}{R+R_{S}}-\frac{\beta}{R \kappa}\right)+ \\
\frac{\beta}{2 R \kappa} \sum_{ \pm}|\omega \pm e V|\left[|\omega \pm e V| R_{0} C\right]^{\frac{2}{g_{0}}}
\end{array}
$$

If both $\omega$ and $V$ tend to zero, the last term in (55) can again be neglected and we find

$$
\delta \mathcal{S}_{\omega}=-\frac{|\omega| \beta}{R \kappa}
$$

If, however, $\omega$ and/or $e V$ exceed the scale $g_{0} E_{C} \exp \left(-g_{0} / 2\right)$, one expands Eq. (55) in $2 / g_{0}$ and gets

$$
\delta \mathcal{S}_{\omega}=-\frac{2|\omega| \beta}{g_{0} R \kappa} \ln \frac{1}{|\omega| R_{0} C} .
$$

This expression applies for $\omega \gg e V$. In the opposite limit in Eq. (57) one should simply substitute $\mathrm{eV}$ instead of $\omega$. Note, however, that in the latter limit the corresponding result yields only one contribution $\left(\delta S_{\omega}^{(\beta)}\right)$ to the interaction correction. Another contribution $\left(\delta S_{\omega}^{(\gamma)}\right)$ will be found in Sec. $3 \mathrm{~F}$.

To complete this subsection let us find the interaction correction in the limit $\omega, e V \gg 1 / R_{0} C$. At large voltages the dependence of $F(t)$ on $V$ should be taken into 
account. Evaluating the corresponding (linear in $V$ ) correction to $F(t)(51)$, we obtain

$$
\delta \mathcal{S}_{\omega}=\frac{e^{2} R_{0}^{2} \beta^{2}|e V|}{4 \pi R^{2} \kappa \Omega} \sum_{ \pm} w\left[(\omega \pm e V) R_{0} C\right]
$$

where

$$
w(x)=-2-2 x \arctan x-\ln x^{2}+\ln \left(1+x^{2}\right)+|x| \pi .
$$

For $|\omega \pm \mathrm{eV}| \gg 1 / R_{0} C$ the asymptotics $w(x \gg 1) \simeq$ $1 / 3 x^{2}$ should be used. In this case we again recover Eq. (49). If, however, $|\omega \pm e V| \lesssim 1 / R_{0} C$, then the interaction correction is governed by another asymptotics $w(x \ll$ $1) \simeq-2-\ln x^{2}$ and, hence, this correction is positive. Such an increase of the noise at $|\omega \pm e V|<1 / R_{0} C$ is similar to that found at higher temperatures.

\section{F. Large voltages}

Now let us evaluate the remaining correction $\delta \mathcal{S}^{(\gamma)}$ (36). At high temperatures $T \gg 1 / C R_{0}$ we obtain

$$
\begin{array}{r}
\delta \mathcal{S}_{\omega}^{(\gamma)} \sim \frac{e^{2} \gamma}{R C \kappa \Omega}\left(\frac{e V}{T}\right)^{2}, \quad \text { if } \omega, e V \ll T, \\
\delta \mathcal{S}_{\omega}^{(\gamma)}=\frac{e^{2} \gamma}{R C \kappa \Omega} \theta(|e V|-|\omega|) \tanh \frac{|e V|-|\omega|}{2 T}, \\
\text { if } \omega, e V \gg T .
\end{array}
$$

In the limit $\omega, e V \ll T \ll 1 / C R_{0}$ one finds

$$
\delta \mathcal{S}_{\omega}^{(\gamma)}=\frac{2 \gamma(e V)^{2}}{3 T g_{0} R \kappa} \ln \frac{1}{T R_{0} C} .
$$

Finally, at higher frequencies and voltages $\omega, e V \gg T$ we derive

$$
\begin{gathered}
\delta \mathcal{S}_{\omega}^{(\gamma)}=\frac{4 \gamma}{g_{0} R \kappa \Omega} \theta(|e V|-|\omega|)\left\{\frac{\arctan \left[(|e V|-|\omega|) R_{0} C\right]}{R_{0} C}\right. \\
\left.+\frac{|e V|-|\omega|}{2} \ln \left[1+\left((|e V|-|\omega|) R_{0} C\right)^{-2}\right]\right\} .
\end{gathered}
$$

Note that the correction $\delta \mathcal{S}_{\omega}^{(\gamma)}$ is positive in all cases. As compared to previously obtained contribution $\delta S_{\omega}^{(\beta)}$ the correction (63) becomes important in the limit $\mathrm{eV} \gg$ $T, \omega$. For such voltages both corrections add up, $\delta \mathcal{S}_{\omega}=$ $\delta \mathcal{S}_{\omega}^{(\beta)}+\delta \mathcal{S}_{\omega}^{(\gamma)}$, and yield

$$
\begin{array}{r}
\delta \mathcal{S}_{\omega}=-\frac{2(\beta-2 \gamma)|e V|}{g_{0} R \kappa} \ln \frac{1}{|e V| R_{0} C}, \\
\text { if } T,|\omega| \ll|e V| \ll 1 / C R_{0}, \\
\delta \mathcal{S}_{\omega}=-\frac{(\beta-2 \gamma) E_{C}}{R \kappa \Omega}, \quad \text { if }|e V| \gg T, 1 / C R_{0},|\omega| .
\end{array}
$$

These results complete our analysis of current fluctuations in coherent conductors with electron-electron interactions.

\section{SUMMARY}

Combining the standard scattering matrix approach with the effective action formalism we have analyzed the effect of electron-electron interactions on current noise in mesoscopic coherent conductors in the metallic limit (7).

We have found that Coulomb interaction always leads to partial suppression of the Nyquist noise. The corresponding interaction term is proportional to the parameter $\beta$ (11) similarly to the interaction correction to the conductance 5 . Interaction-induced suppression of both conductance and Nyquist noise has the same physical origin, and a direct relation between these two effects can easily be established with the aid of FDT.

The effect of electron-electron interactions on the shot noise is somewhat more complicated. In this case we have recovered two different interaction corrections entering with opposite signs. One of them is negative and it is again governed by the parameter $\beta$. Another correction is positive and it is proportional to the parameter $\gamma$ (4) which depends on the transmission distribution in a different way. The net interaction correction to the shot noise scales as $\delta \mathcal{S}_{\omega}(V) \propto 2 \gamma-\beta$, i.e. it can be both negative and positive depending on the relation between $\beta$ and $\gamma$. The contribution to $\delta \mathcal{S}_{\omega}(V)$ from the $n$-th conducting mode is negative provided its transmission $T_{n}$ is smaller than $1 / 2$ and it is positive otherwise. For coherent diffusive conductors $2 \gamma-\beta=-1 / 15$, i.e. in this particular case electron-electron interactions tend to decrease the shot noise.

The presence of two interaction corrections to the shot noise has a transparent physical interpretation. The $\beta$ correction is due to Coulomb blockade suppression of the Landauer conductance (2) while the $\gamma$-correction originates from the term $-\sum_{n} T_{n}^{2}$ in the expression for the shot noise2, 3. 4 . The absolute value of this term is also decreased by interactions. But, since it enters with the negative sign, the corresponding contribution to the noise spectrum turns out to be positive. We believe that the effect of electron-electron interactions on higher cumulants of the current operator can be described in a similar manner.

This work is part of the Kompetenznetz " Funktionelle Nanostructuren" supported by the Landestiftung BadenWürttemberg gGmbH. One of us (A.V.G.) acknowledges support from the Alexander von Humboldt Stiftung. 


\section{APPENDIX A: EFFECTIVE ACTION AND OBSERVABLES}

Following 3 let us combine the effective action formalism 18.19 with the usual Landauer scattering approach. Within the latter approach one introduces a (relatively small) scatterer which connects two bulk reservoirs. The scatterer is described by the scattering matrix. In order to include electron-electron interactions it is necessary to reckon with the many-body Hamiltonian

$$
\hat{H}=\int d \boldsymbol{r} \hat{\Psi}^{+}(\boldsymbol{r})\left[-\frac{\nabla^{2}}{2 m}+W(\boldsymbol{r})\right] \hat{\Psi}(\boldsymbol{r})+\frac{1}{2} \int d \boldsymbol{r} \int d \boldsymbol{r}^{\prime} \hat{\Psi}^{+}(\boldsymbol{r}) \hat{\Psi}^{+}\left(\boldsymbol{r}^{\prime}\right) \frac{e^{2}}{\left|\boldsymbol{r}-\boldsymbol{r}^{\prime}\right|} \Psi\left(\boldsymbol{r}^{\prime}\right) \Psi(\boldsymbol{r}) .
$$

Here the term $W(\boldsymbol{r})$ accounts for boundary and impurity potentials, external fields etc. After the standard HubbardStratonovich decoupling of the interaction term one arrives at the following path integral over an auxiliary field $V(\boldsymbol{r}, t)$

$$
e^{-i \hat{H} t}=\frac{\int \mathcal{D} V\left(\boldsymbol{r}, t^{\prime}\right)\left[\hat{\mathbf{T}} e^{-i \int_{0}^{t} d t^{\prime} \hat{H}_{\mathrm{eff}}\left[V\left(\boldsymbol{r}, t^{\prime}\right)\right]}\right] e^{i \int_{0}^{t} d t^{\prime} \int d \boldsymbol{r} \frac{\nabla V\left(\boldsymbol{r}, t^{\prime}\right)^{2}}{8 \pi}}}{\int \mathcal{D} V\left(\boldsymbol{r}, t^{\prime}\right) e^{i \int_{0}^{t} d t^{\prime} \int d \boldsymbol{r} \frac{\nabla V\left(\boldsymbol{r}, t^{\prime}\right)^{2}}{8 \pi}}} .
$$

Here $\hat{\mathbf{T}}$ is the time-ordering operator and

$$
\hat{H}_{e f f}\left[V\left(\boldsymbol{r}, t^{\prime}\right)\right]=\int d \boldsymbol{r} \hat{\Psi}^{+}(\boldsymbol{r})\left[-\frac{\nabla^{2}}{2 m}+W(\boldsymbol{r})-e V(\boldsymbol{r}, t)\right] \hat{\Psi}(\boldsymbol{r}) .
$$

We choose to define the electron charge as $-e$.

The time dynamics of the density matrix $\rho$ is described by means of the evolution operator $J$ defined on the Keldysh contour. In what follows we shall denote the field $V$ on the upper and lower parts of this contour by $V_{1,2}$. The general expression for the density matrix reads

$$
\rho\left(X_{1 f}, X_{2 f}, t_{f}\right)=\int d X_{1 i} d X_{2 i} J\left(X_{1 f}, X_{2 f} ; X_{1 i}, X_{2 i} ; t_{f}, t_{i}\right) \rho\left(X_{1 i}, X_{2 i}, t_{i}\right),
$$

where $X$ the set of relevant quantum degrees of freedom. We shall assume that interaction with the fluctuating fields $V_{1,2}$ is turned on at a time $t_{i}=0$. The time $t_{f}$ is supposed to be large. Making use of (A2) and integrating over the fermionic degrees of freedom, we obtain

$$
J=\int \mathcal{D} V_{1} \mathcal{D} V_{2} \exp i S\left[V_{1}, V_{2}\right]
$$

where $S$ is the effective action

$$
i S\left[V_{1}, V_{2}\right]=2 \operatorname{Tr} \ln \hat{G}_{V}^{-1}+i \frac{C}{2} \int_{0}^{t} d t^{\prime}\left[V_{L R 1}^{2}-V_{L R 2}^{2}\right],
$$

Here we defined $V_{L R j}=V_{L j}-V_{R j}$ and neglected the spatial dependence of the fields $V_{L 1,2}$ and $V_{R 1,2}$ inside both the left $(\mathrm{L})$ and the right $(\mathrm{R})$ reservoirs. The Green-Keldysh matrix $G_{V}\left(X_{1}, X_{2}\right)$ (here $X=(\boldsymbol{r}, t)$ ) obeys the $2 \times 2$ matrix equation

$$
\left(i \frac{\partial}{\partial t_{1}} \hat{\mathbf{1}}-\hat{H}_{0}\left(\mathbf{r}_{1}\right) \hat{\mathbf{1}}+e \hat{V}\left(X_{1}\right)\right) \hat{G}_{V}\left(X_{1}, X_{2}\right)=\delta\left(X_{1}-X_{2}\right) \hat{\sigma}_{z}
$$

where $\hat{H}_{0}=\left(-\nabla^{2} / 2 m\right)+W(\boldsymbol{r}), \hat{V}$ is a diagonal $2 \times 2$ matrix with components $\hat{V}_{i j}=V_{i} \delta_{i j}$ and $\hat{\sigma}_{z}$ is the Pauli matrix.

The above equation for the Green-Keldysh function should be supplemented by the initial condition for the density matrix $\hat{\rho}(t=0)=\hat{\rho}_{0}$, where $\hat{\rho}_{0}$ is the equilibrium density matrix of noninteracting electrons. In what follows we will need the solution of Eq. (A7) in the absence of the fulcutating fields $V_{1,2}$, which reads

$$
\begin{aligned}
& G_{11}\left(t_{1}, t_{2}\right)=-i \theta\left(t_{1}-t_{2}\right) \hat{U}_{1}\left(t_{1}, t_{2}\right)+i \hat{U}_{1}\left(t_{1}, 0\right) \hat{\rho}_{0} \hat{U}_{1}\left(0, t_{2}\right), \\
& G_{22}\left(t_{1}, t_{2}\right)=-i \theta\left(t_{2}-t_{1}\right) \hat{U}_{2}\left(t_{1}, t_{2}\right)+i \hat{U}_{2}\left(t_{1}, 0\right) \hat{\rho}_{0} \hat{U}_{2}\left(0, t_{2}\right), \\
& G_{12}\left(t_{1}, t_{2}\right)=i \hat{U}_{1}\left(t_{1}, 0\right) \hat{\rho}_{0} \hat{U}_{2}\left(0, t_{2}\right), \\
& G_{21}\left(t_{1}, t_{2}\right)=-i \hat{U}_{2}\left(t_{1}, 0\right)\left[\hat{1}-\hat{\rho}_{0}\right] \hat{U}_{1}\left(0, t_{2}\right),
\end{aligned}
$$


where $\hat{U}_{1,2}\left(t_{1}, t_{2}\right)$ are the evolution operators

$$
\hat{U}_{1,2}\left(t_{1}, t_{2}\right)=\mathrm{T} \exp \left[-i \int_{t_{2}}^{t_{1}} d t^{\prime}\left(\hat{H}_{0}-e V_{1,2}\left(\boldsymbol{r}, t^{\prime}\right)\right)\right] .
$$

One should keep in mind that in the operator products like $\hat{U} \hat{\rho} \hat{U}$ integration over intermediate spatial coordinates is implied.

Instead of specifying $W(\boldsymbol{r})$ we will describe electron transfer between the reservoirs by means of the scattering matrix formalism. This procedure is standard and further details are provided in Appendix B. In calculating the trace in Eq.(A6), we shall make an explicit integration over the longitudinal coordinates. Integration over the transverse coordinates is replaced by summing over the transmission channels of the scatterer. It is convenient to introduce the phase variables

$$
\begin{gathered}
\varphi^{+}(t)=\int_{0}^{t} d t^{\prime}\left(e V_{L R 1}\left(t^{\prime}\right)+e V_{L R 2}\left(t^{\prime}\right)\right) / 2, \\
\varphi^{-}(t)=\int_{0}^{t} d t^{\prime}\left(e V_{L R 1}\left(t^{\prime}\right)-e V_{L R 2}\left(t^{\prime}\right)\right) .
\end{gathered}
$$

Provided the dimensionless conductance $g_{0}$ is large $g \gg 1$ fluctuations of $\varphi^{-}(t)$ are strongly suppressed, so we can expand the exact action $i S\left[\varphi^{ \pm}\right]$in powers of $\varphi^{-}$keeping the full nonlinearity in $\varphi^{+}$. Note, that the external voltage enters only in $\varphi^{+}$but not in $\varphi^{-}$. Hence, for the system of Fig. 1 we have to impose the constraints $\sum_{j} \varphi_{j}^{+}(t)=e V_{x} t$, $\sum_{j} \varphi_{j}^{-}(t)=0$. Here and below in this Appendix the summation runs over different elements in our circuit, i.e. the scatterer and the shunt.

Let us define the kernel of the current operator. Consider, e.g., the upper part of the Keldysh contour. The charge is proportional to the derivative of the action with the respect to the external voltage. Due to the property $\delta S / \delta V_{1}(\boldsymbol{r}, t)=e \int_{0}^{t} \delta S / \delta \varphi_{1}(\boldsymbol{r}, t)$ we can relate the difference of the charges of the left and right reservoirs to the integral of $\delta S / \delta \varphi_{L R}(t)$ over time. Hence, the latter derivative is proportional to the current through the scatterer. Keeping track of proper ordering in the upper part of the Keldysh contour, for $t>t^{\prime}$ we obtain

$$
\left\langle\hat{I}_{j}(t) \hat{I}_{j}\left(t^{\prime}\right)\right\rangle=-e^{2} \int \mathcal{D} \varphi^{ \pm} \frac{\delta^{2}}{\delta \varphi_{1 j}(t) \delta \varphi_{1 j}\left(t^{\prime}\right)} e^{i S\left[\varphi^{ \pm}\right]} .
$$

Combining (A12) with a similar relation in the lower part of the Keldysh contour we get

$$
\frac{1}{2}\left\langle\hat{I}_{j}(t) \hat{I}_{j}\left(t^{\prime}\right)+\hat{I}_{j}\left(t^{\prime}\right) \hat{I}_{j}(t)\right\rangle=-\frac{e^{2}}{2} \int \mathcal{D} \varphi^{ \pm}\left[\frac{\delta^{2}}{\delta \varphi_{1 j}(t) \delta \varphi_{1 j}\left(t^{\prime}\right)}+\frac{\delta^{2}}{\delta \varphi_{2 j}(t) \delta \varphi_{2 j}\left(t^{\prime}\right)}\right] e^{i S\left[\varphi^{ \pm}\right]} .
$$

This equation is equivalent to (18).

Below we shall proceed with an explicit calculation of the action by defining the scattering states.

\section{APPENDIX B: TRANSMISSION CHANNELS}

Following the usual $\hat{S}$-matrix approach 20 let us introduce the transmission channels. We will assume that far from the scatterer the electron propagation in transverse and longitudinal directions can be described separately. In this case the solution of the Schrödinger equation

$$
-\frac{\nabla^{2}}{2 m} \psi(\boldsymbol{r})+W(\boldsymbol{r}) \psi(\boldsymbol{r})=E \psi(\boldsymbol{r})
$$

can be factorized

$$
\psi(\boldsymbol{r})=\sum_{n} c_{n} \Phi_{n}\left(\boldsymbol{r}_{\perp}\right) \chi_{n}(x) .
$$

Here $x$ is the coordinate along the lead and $\boldsymbol{r}_{\perp}$ are the transverse coordinates. The transverse wave functions $\Phi_{n}\left(\boldsymbol{r}_{\perp}\right)$ satisfy the equation

$$
-\frac{\nabla_{\perp}^{2}}{2 m} \Phi_{n}\left(\boldsymbol{r}_{\perp}\right)+W\left(\boldsymbol{r}_{\perp}\right) \Phi_{n}\left(\boldsymbol{r}_{\perp}\right)=E_{n} \Phi\left(\boldsymbol{r}_{\perp}\right)
$$




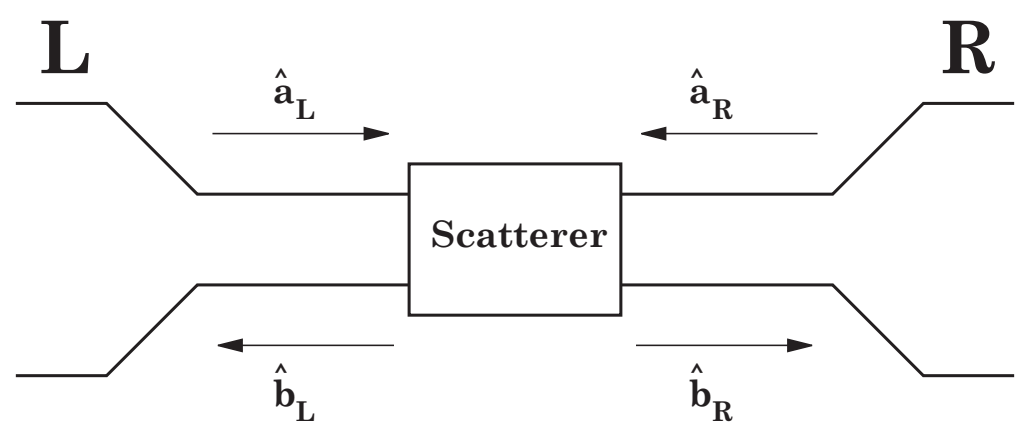

FIG. 2: Scattering states

where the subscript $n$ enumerates the transmission channels (we are considering only channels with $E_{n}<E_{F}$ ). The function $\chi_{n}(x)$ outside the scatterer region is defined from the equation

$$
-\frac{1}{2 m} \frac{d^{2}}{d x^{2}} \chi_{n}(x)=\left(E-E_{n}\right) \chi_{n}(x) .
$$

Since the electronic states with energies $E$ close to the Fermi energy $E_{F}$ mainly contribute, it is sufficient to describe the electron dynamics quasiclassically. We define the energy $\xi=E-E_{F}$ and the particle velocity in the $n$-th channel $v_{n}=\sqrt{2\left(E_{F}-E_{n}\right) / m}$. Then the wave function can be expressed as

$$
\begin{array}{ll}
\chi_{n}(x)=e^{i m v_{n} x} f_{n}^{i n}(x)+e^{-i m v_{n} x} f_{n}^{\text {out }}(x), & \text { left reservoir. } \\
\chi_{n}(x)=e^{i m v_{n} x} g_{n}^{\text {out }}(x)+e^{-i m v_{n} x} g_{n}^{\text {in }}(x), & \text { right reservoir. }
\end{array}
$$

In this way we have introduced the envelopes of the fast oscillating functions $\exp \left( \pm i m v_{n} x\right)$. Consider first the left reservoir. The functions $f_{n}^{i n}(x)$ and $f_{n}^{\text {out }}(x)$ satisfy the following quasiclassical equations

$$
\begin{aligned}
-i v_{n} \frac{d}{d x} f_{n}^{i n}(x) & =\xi f_{n}^{i n}(x), \\
i v_{n} \frac{d}{d x} f_{n}^{\text {out }}(x) & =\xi f_{n}^{\text {out }}(x)
\end{aligned}
$$

with the solutions

$$
f_{n}^{i n}(x)=\frac{e^{i \xi x / v_{n}}}{\sqrt{v_{n}}}, \quad f_{n}^{o u t}(x)=\frac{e^{-i \xi x / v_{n}}}{\sqrt{v_{n}}} .
$$

Analogously, for the right reservoir we find

$$
g_{m}^{i n}(x)=\frac{e^{-i \xi x / v_{m}}}{\sqrt{v_{m}}}, \quad g_{n}^{\text {out }}(x)=\frac{e^{i \xi x / v_{m}}}{\sqrt{v_{m}}} .
$$

The eigenfunction of the whole system with the energy $\xi$ in the left reservoir may be expressed as

$$
\psi_{\xi}(\boldsymbol{r})=\sum_{n}\left[a_{L n} e^{i m v_{n} x} f_{n}^{i n}(x)+b_{L n} e^{-i m v_{n} x} f_{n}^{\text {out }}(x)\right] \Phi_{n}\left(\boldsymbol{r}_{\perp}\right),
$$

while in the right reservoir we get

$$
\psi_{\xi}(\boldsymbol{r})=\sum_{k}\left[b_{R k} e^{i m v_{k} x} g_{k}^{o u t}(x)+a_{R k} e^{-i m v_{k} x} g_{k}^{i n}(x)\right] \Phi_{k}\left(\boldsymbol{r}_{\perp}\right) .
$$

The amplitudes of the outgoing $b_{L, R}$ and incoming $a_{L, R}$ states (see the Fig. 2) are related via the scattering matrix $\hat{S}(\xi)$

$$
\left(\begin{array}{c}
b_{L 1} \\
\cdots \\
b_{L N_{L}} \\
b_{R 1} \\
\cdots \\
b_{R N_{R}}
\end{array}\right)=\hat{S}(\xi)\left(\begin{array}{c}
a_{L 1} \\
\cdots \\
a_{L N_{L}} \\
a_{R 1} \\
\cdots \\
a_{R N_{R}}
\end{array}\right) .
$$


The unitary matrix $\hat{S}$ with dimensions $\left(N_{L}+N_{R}\right) \times\left(N_{L}+N_{R}\right)$ has the block structure

$$
\hat{S}(\xi)=\left(\begin{array}{cc}
\hat{r}(\xi) & \hat{t}^{\prime}(\xi) \\
\hat{t}(\xi) & \hat{r}^{\prime}(\xi)
\end{array}\right)
$$

The diagonal blocks $\hat{r}$ and $\hat{r}^{\prime}$ describe reflection back to the left and right reservoirs, respectively. The off-diagonal blocks describe transmission through the scatterer. Later we shall neglect the $\xi$-dependence of $\hat{S}$.

Let us now combine the incident $f_{n}^{\text {in }}(x)$ and outgoing $f_{n}^{\text {out }}(x)$ wave functions belonging to the same channel into one wave function $\psi_{n}(x)$. Namely, we assume that the scatterer is located at $x=0$, and for the left reservoir $(x<0)$ we put

$$
\psi_{n}(y)= \begin{cases}f_{n}^{\text {in }}(y), & y<0 \\ f_{n}^{\text {out }}(-y), & y>0\end{cases}
$$

Analogously, for the right reservoir $(x>0)$ we define

$$
\psi_{m}(y)= \begin{cases}f_{m}^{\text {in }}(-y), & y<0 \\ f_{m}^{\text {out }}(y), & y>0\end{cases}
$$

These new functions are defined in all the range $y \in[-\infty,+\infty]$ and are equal to

$$
\psi_{j}(y)=\frac{e^{i \xi y / v_{j}}}{\sqrt{v_{j}}} .
$$

Let us emphasize, that here the index $j$ enumerates all conducting channels, both in the left and in the right reservoirs (for convenience, we assume that the left channels are enumerated first).

In the presence of the fluctuating field $V(t)$, the matrix elements of the Hamiltonian in the reservoirs take the form

$$
\hat{H}_{i j}=-i v_{i} \delta_{i j} \frac{\partial}{\partial y}-e V_{i}(t) \delta_{i j},
$$

where $V_{i}=V_{L}$ for all left channels and $V_{i}=V_{R}$ for the right channels. If at initial time $t_{1}$ the wave function was $\psi_{n}\left(t_{1}, y\right)$, then at the final time $t_{2}>t_{1}$ it becomes

$$
\begin{aligned}
& \psi_{n}\left(t_{2}, y\right)=e^{i\left[\varphi_{n}\left(t_{2}\right)-\varphi_{n}\left(t_{1}\right)\right]} \psi_{n}\left(t_{1}, y-v_{n}\left(t_{2}-t_{1}\right)\right), y<0 \text { or } y>v_{n}\left(t_{2}-t_{1}\right) \\
& \psi_{n}\left(t_{2}, y\right)=\sum_{k} e^{i\left[\varphi_{n}\left(t_{2}\right)-\varphi_{k}\left(t_{1}\right)\right]-i\left[\varphi_{n}\left(t_{2}-\frac{y}{v_{n}}\right)-\varphi_{k}\left(t_{2}-\frac{y}{v_{n}}\right)\right]} s_{n k} \sqrt{\frac{v_{k}}{v_{n}}} \psi_{k}\left(t_{1}, \frac{v_{k}}{v_{n}} y-v_{k}\left(t_{2}-t_{1}\right)\right), 0<y<v_{n}\left(t_{2}-t_{1}\right) .
\end{aligned}
$$

Here $s_{n k}$ are the matrix elements of the $\hat{S}$-matrix and, as before, $\varphi_{n}(t)=\int_{0}^{t} d \tilde{t} e V_{n}(\tilde{t})$. On the other hand, by definition of the evolution operator we have

$$
\psi_{n}\left(t_{2}, y_{2}\right)=\sum_{k} \int d y_{1} U_{n k}\left(t_{2}, t_{1} ; y_{2}, y_{1}\right) \psi_{k}\left(t_{1}, y_{1}\right)
$$

Comparing this expression with (B17), we find

$$
\begin{aligned}
U_{n k}\left(t_{2}, t_{1} ; y_{2}, y_{1}\right)= & e^{i \varphi_{n}\left(t_{2}\right)}\left\{\frac{\delta_{n k}}{v_{n}} \delta\left(\frac{y_{2}-y_{1}}{v_{n}}-t_{2}+t_{1}\right)+\theta\left(y_{2}\right) \theta\left(v_{n}\left(t_{2}-t_{1}\right)-y_{2}\right) e^{-i \varphi_{n}\left(t_{2}-\frac{y_{2}}{v_{n}}\right)\left[s_{n k}-\delta_{n k}\right] \times}\right. \\
& \left.\sqrt{\frac{v_{k}}{v_{n}}} e^{i \varphi_{k}\left(t_{2}-\frac{y_{2}}{v_{n}}\right)} \delta\left(\frac{v_{k}}{v_{n}} y_{2}-y_{1}-v_{k}\left(t_{2}-t_{1}\right)\right)\right\} e^{-i \varphi_{k}\left(t_{1}\right)} .
\end{aligned}
$$

It is convenient to introduce the new coordinates $\tau=y / v_{n}$. More precisely, instead of the wave function with the components $\psi_{n}(y)$ we introduce the functions $\eta_{n}(\tau)=\sqrt{v_{n}} \psi_{n}\left(y / v_{n}\right)$. The kernels of the operators will also be transformed. If the two functions are related to each other by means of a linear operator

$$
\psi_{n}^{(2)}(y)=\sum_{k} \int d y^{\prime} K_{n k}\left(y, y^{\prime}\right) \psi_{k}^{(1)}\left(y^{\prime}\right),
$$


then the corresponding wave functions $\eta^{(2)}$ and $\eta^{(1)}$ satisfy the following relation:

$$
\eta_{n}^{(2)}(\tau)=\sum_{k} \int d \tau^{\prime} \tilde{K}_{n k}\left(\tau, \tau^{\prime}\right) \eta_{k}^{(1)}\left(\tau^{\prime}\right)
$$

where

$$
\tilde{K}_{n k}\left(\tau, \tau^{\prime}\right)=\sqrt{v_{n}} K_{n k}\left(v_{n} \tau, v_{k} \tau^{\prime}\right) \sqrt{v_{k}} .
$$

In this representation the evolution operator can be simplified. We find

$$
\hat{U}\left(t_{2}, t_{1} ; \tau_{2}, \tau_{1}\right)=\delta\left(\tau_{2}-\tau_{1}-t_{2}+t_{1}\right) e^{i \hat{\varphi}\left(t_{2}\right)}\left\{\hat{1}+\theta\left(\tau_{2}\right) \theta\left(-\tau_{1}\right) e^{-i \hat{\varphi}\left(t_{2}-\tau_{2}\right)}[\hat{S}-\hat{1}] e^{i \hat{\varphi}\left(t_{1}-\tau_{1}\right)}\right\} e^{-i \hat{\varphi}\left(t_{1}\right)} .
$$

The matrix $\hat{\varphi}$ is diagonal with respect to the channel indices $\hat{\varphi}_{i k}=\varphi_{i} \delta_{i k}$. We also obtain an expression for the inverse operator, i.e. the operator defined by $\int d \tau_{2} \hat{U}\left(t_{2} t_{1} ; \tau_{3} \tau_{2}\right) \hat{U}^{-1}\left(t_{2} t_{1} ; \tau_{2} \tau_{1}\right)=\delta\left(\tau_{3}-\tau_{1}\right)$. It reads

$$
\hat{U}^{-1}\left(t_{2}, t_{1} ; \tau_{2}, \tau_{1}\right)=\delta\left(\tau_{1}-\tau_{2}-t_{2}+t_{1}\right) e^{i \hat{\varphi}\left(t_{1}\right)}\left\{\hat{1}+\theta\left(\tau_{1}\right) \theta\left(-\tau_{2}\right) e^{-i \hat{\varphi}\left(t_{1}-\tau_{2}\right)}\left[\hat{S}^{+}-\hat{1}\right] e^{i \hat{\varphi}\left(t_{2}-\tau_{1}\right)}\right\} e^{-i \hat{\varphi}\left(t_{2}\right)}
$$

Eqs. (B21), (B22) apply for $t_{2}>t_{1}$, in order to construct the corresponding expressions in the opposite case one should just use the property $\hat{U}\left(t_{2}, t_{1} ; \tau_{2}, \tau_{1}\right)=\hat{U}^{-1}\left(t_{1}, t_{2} ; \tau_{2}, \tau_{1}\right)$.

Finally let us define the equilibrium density matrix for noninteracting electrons. It can be written in the form

$$
\rho_{0, n k}\left(y_{1}, y_{2}\right)=\delta_{n k} \int \frac{d p}{2 \pi} \frac{e^{i p\left(y_{1}-y_{2}\right)}}{1+e^{p v_{n} / T}}=\frac{\delta_{n k}}{2} \delta\left(y_{1}-y_{2}\right)-\frac{\delta_{n k}}{2 \pi} \frac{\pi i T}{v_{n} \sinh \left[\frac{\pi T\left(y_{1}-y_{2}\right)}{v_{n}}\right]}
$$

Performing the transformation (B20) we obtain

$$
\hat{\rho}_{0}\left(\tau_{1}, \tau_{2}\right)=\frac{1}{2}\left(\delta\left(\tau_{1}-\tau_{2}\right)-\frac{i T}{\sinh \left[\pi T\left(\tau_{1}-\tau_{2}\right)\right]}\right) \hat{1} .
$$

\section{APPENDIX C: EXPANSION IN THE PHASE DIFFERENCE}

We shall expand the effective action $(\sqrt{\mathrm{A} 6})$ perturbatively in $\varphi^{-}$. The field $\varphi^{+}$will be taken into account exactly in each term of this expansion. The expansion starts from the first order in $\varphi^{-}$, since for $\varphi^{-}=0$ the contributions from the forward and backward parts of the Keldysh contour cancel each other. We get from Eq. A7)

$$
2 \operatorname{Tr} \ln \hat{G}_{V}^{-1}=2 \operatorname{Tr} \ln \left(1+\frac{\hat{G}_{0} \hat{\dot{\varphi}}^{-}}{2}\right) .
$$

The Green-Keldysh matrix $\hat{G}_{0}$ is evaluated for $\hat{\varphi}^{-}=0$, i.e. it is defined by Eqs. (A8) with the evolution operator (B21) taken at $\hat{\varphi}=\hat{\varphi}^{+}$). The fluctuating field $\hat{\varphi}^{-}$in (C1) is a unity matrix in Keldysh space and a diagonal matrix in the space of conducting channels. Performing the summation over the Keldysh indices we arrive at the first order in $\varphi^{-}$contribution to the action $i S^{(1)}$

$$
i S^{(1)}\left[\varphi^{ \pm}\right]=-i \int d t \int d s \int d \tau_{1} \int d \tau_{2} \operatorname{Tr}\left[\hat{U}\left(t, 0 ; s, \tau_{1}\right)\left\{\frac{1}{\pi} \frac{\pi i T}{\sinh \left[\pi T\left(\tau_{1}-\tau_{2}\right)\right]}\right\} \hat{U}^{-1}\left(t, 0 ; \tau_{2}, s\right) \hat{\dot{\varphi}}^{-}(t)\right] .
$$

For simplicity in Sec. 3 we have set $t_{f} \rightarrow \infty$. Here we will keep it finite and use the conditions $\varphi^{-}(0)=\varphi^{-}\left(t_{f}\right)=0$. The $\delta$-functions contained in $\hat{U}$-matrices of (C2) will lead to a singularity of the form $1 / \sinh \left[\pi T\left(\tau_{1}-\tau_{2}\right)\right]$ which is cured as follows. Let us choose close but not exactly equal arguments $s_{1}$ and $s_{2}$. Expanding the combination

$$
-i \int d t \int d \tau_{1} \int d \tau_{2} \operatorname{Tr}\left[\hat{U}\left(t, 0 ; s_{1}, \tau_{1}\right) \hat{U}^{-1}\left(t, 0 ; \tau_{2}, s_{2}\right) \hat{\dot{\varphi}}^{-}(t)\right]
$$

to the first order in $s_{1}-s_{2}$ and multiplying the result by $1 / \sinh \left[\pi T\left(s_{1}-s_{2}\right)\right]$, we obtain

$$
i S^{(1)}\left[\varphi^{ \pm}\right]=\frac{i}{\pi} \int_{0}^{t_{f}} d s \operatorname{Tr}\left[\hat{\varphi}^{-}(s)\left(\hat{S}_{\hat{\varphi}^{+}}(s) \hat{S}^{+}-\hat{\dot{\varphi}}^{+}(s)\right)\right] .
$$


Making use of the condition $\operatorname{Tr}\left[\hat{t}^{+} \hat{t}\right]=\operatorname{Tr}\left[\hat{t}^{\prime} \hat{t}^{\prime}\right]$ we get

$$
i S^{(1)}\left[\varphi^{ \pm}\right]=-\frac{i}{\pi} \operatorname{Tr}\left[\hat{t}^{+} \hat{t}\right] \int_{0}^{t_{f}} d \tau \varphi^{-}(\tau) \dot{\varphi}^{+}(\tau)
$$

where $\varphi^{ \pm}(\tau)=\varphi_{L}^{ \pm}(\tau)-\varphi_{R}^{ \pm}(\tau)$.

Consider now the contribution to the action of the second order in $\varphi^{-}$. It is defined as

$$
i S^{(2)}\left[\varphi^{ \pm}\right]=-\operatorname{Tr}\left[\hat{G}_{12} \hat{\dot{\varphi}}^{-} \hat{G}_{21} \hat{\dot{\varphi}}^{-}\right]
$$

After a straightforward algebra one obtains

$$
\begin{aligned}
i S^{(2)}\left[\varphi^{ \pm}\right]=- & \int_{0}^{t_{f}} d \tau_{1} \int_{0}^{t_{f}} d \tau_{2} \rho_{0}\left(\tau_{2}-\tau_{1}\right) \rho_{0}^{*}\left(\tau_{1}-\tau_{2}\right) \times \\
& \operatorname{Tr}\left\{e^{-i\left[\hat{\varphi}^{+}\left(\tau_{1}\right)-\hat{\varphi}^{+}\left(\tau_{2}\right)\right]}\left[\hat{S}^{+} \hat{\varphi}^{-}\left(\tau_{1}\right) \hat{S}-\hat{\varphi}^{-}\left(\tau_{1}\right)\right] e^{\left.i\left[\hat{\varphi}^{+}\left(\tau_{1}\right)-\hat{\varphi}^{+}\left(\tau_{2}\right)\right]\left[\hat{S}^{+} \hat{\varphi}^{-}\left(\tau_{2}\right) \hat{S}-\hat{\varphi}^{-}\left(\tau_{2}\right)\right]\right\} .}\right.
\end{aligned}
$$

Taking into account the block structure of the $\hat{S}$-matrix, we find

$$
\begin{aligned}
i S^{(2)}\left[\varphi^{ \pm}\right]= & -\frac{1}{4 \pi} \int_{0}^{t_{f}} d \tau_{1} \int_{0}^{t_{f}} d \tau_{2} \alpha\left(\tau_{1}-\tau_{2}\right)\left\{\left[\operatorname{Tr}\left(\hat{t}^{\prime} \hat{t}^{\prime}\right)^{2}+\operatorname{Tr}\left(\hat{t}^{+} \hat{t}\right)^{2}\right] \varphi^{-}\left(\tau_{1}\right) \varphi^{-}\left(\tau_{2}\right)+\right. \\
& \left.2 \operatorname{Tr}\left[\hat{r}^{\prime}{\hat{r^{\prime}}}^{+} \hat{t}^{+}\right] \cos \left(\varphi^{+}\left(\tau_{1}\right)-\varphi^{+}\left(\tau_{2}\right)\right) \varphi^{-}\left(\tau_{1}\right) \varphi^{-}\left(\tau_{2}\right)\right\},
\end{aligned}
$$

where $\alpha(\tau)$ is defined in Eq. (21). Introducing the parameter $\beta=\operatorname{Tr}\left[\hat{r}^{\prime} \hat{r}^{\prime}+\hat{t} \hat{t}^{+}\right] / \operatorname{Tr}\left[\hat{t} \hat{t}^{+}\right]$we rewrite Eq. (C8) in a more compact form

$$
i S^{(2)}\left[\varphi^{ \pm}\right]=-\frac{\operatorname{Tr}\left[\hat{t}^{+} \hat{t}\right]}{2 \pi} \int_{0}^{t_{f}} d \tau_{1} \int_{0}^{t_{f}} d \tau_{2} \alpha\left(\tau_{1}-\tau_{2}\right) \varphi^{-}\left(\tau_{1}\right) \varphi^{-}\left(\tau_{2}\right)\left[1-\beta+\beta \cos \left(\varphi^{+}\left(\tau_{1}\right)-\varphi^{+}\left(\tau_{2}\right)\right)\right] .
$$

We now proceed to the third order contribution $i S^{(3)}$ to the effective action. It reads

$$
i S^{(3)}=\frac{i}{2} \operatorname{Tr}\left[\int_{0}^{t_{f}} d t_{1} \int_{0}^{t_{f}} d t_{2} \int_{0}^{t_{f}} d t_{3} \theta\left(t_{1}-t_{2}\right) \theta\left(t_{3}-t_{2}\right) \hat{\mathcal{F}}\left(t_{1}\right) \hat{\mathcal{F}}\left(t_{2}\right) \hat{\mathcal{F}}\left(t_{3}\right) \hat{\rho}_{0}^{a s}\right]+\frac{1}{12} \operatorname{Tr}\left[\left(\hat{G}_{12} \hat{\dot{\varphi}}^{-}+\hat{G}_{21} \hat{\dot{\varphi}}^{-}\right)^{3}\right] .
$$

Here we used the notations

$$
\hat{\rho}_{0}^{a s}\left(\tau_{1}-\tau_{2}\right)=-\frac{i T}{2 \sinh \left[\pi T\left(\tau_{1}-\tau_{2}\right)\right]} \hat{1}, \quad \hat{\mathcal{F}}(t)=\hat{U}^{-1}(t, 0) \hat{\dot{\varphi}}^{-}(t) \hat{U}(t, 0) .
$$

We obtain two terms from Eq. C10. The first one is

$$
i S_{\beta}^{(3)}\left[\varphi^{ \pm}\right]=\frac{i \beta}{6 \pi} \operatorname{Tr}\left[\hat{t}^{+} \hat{t}\right] \int_{0}^{t_{f}} d \tau\left(\varphi^{-}(\tau)\right)^{3} \dot{\varphi}^{+}(\tau) .
$$

In deriving this result we employed the same - although somewhat more involved - regularization procedure as for the first order contribution $S^{(1)}$. This procedure allows to determine the correct overall prefactor in (C12). One can then verify that the resulting effective action satisfies the requirements of FDT.

The second term, coming from Eq. (C10), has the form

$$
\begin{gathered}
i S_{\gamma}^{(3)}\left[\varphi^{ \pm}\right]=\frac{4}{3} \operatorname{Tr}\left[\left(\hat{t} \hat{t}^{+}\right)^{2} \hat{r}^{\prime} \hat{r}^{\prime}\right] \int_{0}^{t_{f}} d y_{1} \int_{0}^{t_{f}} d y_{2} \int_{0}^{t_{f}} d y_{3} \rho_{0}^{a s}\left(y_{2}-y_{1}\right) \rho_{0}^{a s}\left(y_{3}-y_{2}\right) \rho_{0}^{a s}\left(y_{1}-y_{3}\right) \times \\
\varphi^{-}\left(y_{1}\right) \varphi^{-}\left(y_{2}\right) \varphi^{-}\left(y_{3}\right)\left\{\sin \left(\varphi^{+}\left(y_{2}\right)-\varphi^{+}\left(y_{1}\right)\right)+\sin \left(\varphi^{+}\left(y_{3}\right)-\varphi^{+}\left(y_{2}\right)\right)+\sin \left(\varphi^{+}\left(y_{1}\right)-\varphi^{+}\left(y_{3}\right)\right)\right\} .
\end{gathered}
$$


Defining the parameter $\gamma=\operatorname{Tr}\left[\left(\hat{t} \hat{t}^{+}\right)^{2} \hat{r}^{\prime} \hat{r}^{+}\right] / \operatorname{Tr}\left[\hat{t} \hat{t}^{+}\right]$and shifting the phase $\varphi^{+}$by $e V$ we obtain Eq. (35). Collecting now all four contributions (C5), (C9), (C12) and (C13) we arrive at the final result for the effective action

$$
S=S^{(1)}+S^{(2)}+S_{\beta}^{(3)}+S_{\gamma}^{(3)} .
$$

This action is valid up to the third order in $\varphi^{-}$, and the variable $\varphi^{+}$is treated exactly in each of the terms in (C14).

It is instructive to compare our results with the AES action 16 derived for tunnel junctions $(\beta \rightarrow 1)$ to all orders in $\varphi^{ \pm}$. Rewriting the action 10 in our notations together with the capacitive term one has

$$
\begin{aligned}
i S_{A E S}= & \frac{4 i}{e^{2} R} \int_{0}^{t_{f}} d t_{1} \int_{0}^{t_{f}} d t_{2} \alpha_{I}\left(t_{1}-t_{2}\right) \theta\left(t_{1}-t_{2}\right) \sin \left(\varphi^{+}\left(t_{1}\right)-\varphi^{+}\left(t_{2}\right)\right) \sin \frac{\varphi^{-}\left(t_{1}\right)}{2} \cos \frac{\varphi^{-}\left(t_{2}\right)}{2} \\
& -\frac{2}{e^{2} R} \int_{0}^{t_{f}} d t_{1} \int_{0}^{t_{f}} d t_{2} \alpha\left(t_{1}-t_{2}\right) \sin \frac{\varphi^{-}\left(t_{1}\right)}{2} \sin \frac{\varphi^{-}\left(t_{2}\right)}{2} \cos \left(\varphi^{+}\left(t_{1}\right)-\varphi^{+}\left(t_{2}\right)\right)-\frac{i}{e^{2}} \int_{0}^{t_{f}} d t C \ddot{\varphi}^{+} \varphi^{-} .
\end{aligned}
$$

Here we denoted $\alpha_{I}\left(t_{1}-t_{2}\right)=\delta^{\prime}\left(t_{1}-t_{2}\right)$. This $\delta$-function should be understood as a smeared one.

Let us expand $(\overline{\mathrm{C} 15})$ in $\varphi^{-}$and compare with our results order by order. The first order terms are exactly the same for both models. The difference between the models shows up in the second order terms, for our model the parameter $\beta$ appears explicitly in the second order contribution ( $\overline{\mathrm{C} 9}$ ). In the limit $\beta \rightarrow 1$ this expression reduces to that obtained from $(\mathrm{C} 15)$. Expanding the action $(\mathrm{C} 15)$ to the third order in $\varphi^{-}$one only recovers the term of the form (C12) with $\beta=1$, while another term (C13) cannot be recovered. Contributions of this nature are not contained in the AES action at all since they are proportional to higher orders of the channel transmission $T_{n}$.

It is worthwhile to point out that a formally exact represpation for the effective action of a coherent scatterer (all orders in $T_{n}$ and all orders in $\varphi^{ \pm}$) can also be derived11,23.1821. However, this formal expression turns out to he quite complicated to deal with in the situation addressed here. For $g=R_{q} / R \gg 1$ and provided instanton effects1 121 can be neglected all necessary information is equally contained in a much simpler form of the effective action derived in the present paper.

We also note that there exists a simple relation between the action derived here and the cumulant generating function describing the full counting statistics of the charge transport in noninteracting coherent conductors22. This relation can be established if one neglects fluctuations of the phase variable, i.e. sets $\varphi^{+}=\mathrm{eVt}$, and chooses $\varphi^{-}$to be time independent. By identifying $\varphi^{-}=-\lambda$ and expanding the generating function $\ln (\chi(\lambda)$ ) (defined in Eq. (37) of Ref. 22) one arrives at the following indentity:

$$
\ln (\chi(\lambda))=\frac{i}{2} S[e V t,-\lambda]
$$

Finally, let us use the AES action $(\overline{\mathrm{C} 15})$ in order to illustrate the importance of the third order in $\varphi^{-}$terms for the calculation of the currect-current correlation functions. Applying Eq. (A13) one gets a contribution stemming from the double differentiation of the term with $\alpha_{I}$ in the action (C15)

$$
\delta \mathcal{S}\left(t, t^{\prime}\right)=-\left\langle\frac{i}{R} \theta\left(t-t^{\prime}\right) \alpha_{I}\left(t-t^{\prime}\right) \sin \left(\varphi^{+}(t)-\varphi^{+}\left(t^{\prime}\right)\right) \sin \frac{\varphi^{-}(t)-\varphi^{-}\left(t^{\prime}\right)}{2}\right\rangle+\left(t \leftrightarrow t^{\prime}\right) .
$$

Evaluating this average with the aid of path integrals one has to keep all nonlinear terms in the pre-exponent. However, the dependence of the cosine term on $\varphi^{+}$in the action in the exponent can be neglected provided $g \gg 1$. Applying the identity $\sin \left(\varphi^{+}(t)-\varphi^{+}\left(t^{\prime}\right)\right)=\sum_{\nu= \pm} e^{i \nu\left[\varphi^{+}(t)-\varphi^{+}\left(t^{\prime}\right)\right]} / 2 i$ we arrive at the following integral

$$
\int \mathcal{D} \varphi^{+} \exp \left\{i \nu\left(\varphi^{+}(t)-\varphi^{+}\left(t^{\prime}\right)\right)-\frac{i}{e^{2}} \int_{0}^{\infty} d \tilde{t} \varphi^{+}(\tilde{t})\left[C \ddot{\varphi}^{-}(\tilde{t})-\frac{\dot{\varphi}^{-}(\tilde{t})}{R}\right]\right\}
$$

which yields the $\delta$-function

$$
\varphi^{-}(\tilde{t})=\frac{2 \pi}{g} \nu\left[\theta(t-\tilde{t})\left(1-e^{-(t-\tilde{t}) / R C}\right)-\theta\left(t^{\prime}-\tilde{t}\right)\left(1-e^{-\left(t^{\prime}-\tilde{t}\right) / R C}\right)\right] .
$$

As it was expected, $\varphi^{-}(\tilde{t})$ is indeed small for $g \gg 1$. Combining the above expressions we arrive at the contri- bution

$$
\delta \mathcal{S}\left(t, t^{\prime}\right)=-e^{2} \delta\left(t-t^{\prime}\right) / 2 R C
$$


The same contribution multiplied by the factor $\beta$ was derived in Sec. 3B from the term $S_{\beta}^{(3)}$ (31). The above analysis makes the significance of the third order in $\varphi^{-}$ terms in the action particularly transparent: The kernel $\alpha_{I}(t)$ introduces the time-derivative of $e^{-t / R C}$ which compensates for an extra smallness $\sim 1 / g$.
1 Ya.M. Blanter and M. Büttiker, Phys. Rep. 336, 1 (2000).

${ }^{2}$ V.A. Khlus, Sov. Phys. JETP 66, 1243 (1987).

3 G. Lesovik, JETP Lett., 49, 592 (1989).

4 M. Büttiker, Phys. Rev. Lett. 65, 2901 (1990).

5 D.S. Golubev and A.D. Zaikin, Phys. Rev. Lett. 86, 4887 (2001).

6 E. Ben-Jacob, E. Mottola, and G. Schön, Phys. Rev. Lett. 51, 2064 (1983).

7 Yu.V. Nazarov, Sov. J. Low Temp. Phys. 16, 422 (1990).

8 A.D. Zaikin, D.S. Golubev, and S.V. Panyukov, Physica B 203, 417 (1994).

9 H. Lee and L.S. Levitov, Phys. Rev. B 53, 7383 (1996).

10 D.B. Gutman and Yu. Gefen, Phys. Rev. B 64, 205317 (2001).

11 Yu.V. Nazarov, Phys. Rev. Lett. 82, 1245 (1999).

12 D.S. Golubev and A.D. Zaikin, Europhys. Lett. 60, 113 (2002).

13 H. Lee, L.S. Levitov, and A.Yu. Yakovets, Phys. Rev. B 51, 4079 (1995).

14 In this paper we neglect instanton effect.11. 12. These ef- fects are fully suppressed for $R_{S}<R_{q}$, while in the opposite case $R_{S}>R_{q}$ they may become significant only at exponentially small energies.

15 G.-L. Ingold and Yu.V. Nazarov, in Single Charge Tunneling, edited by H. Grabert and M. Devoret (Plenum, New York, 1992), Chap. 2.

16 U. Eckern, G. Schön, and V. Ambegaokar, Phys. Rev. B 30, 6419 (1984).

17 D.S. Golubev, and A.D. Zaikin, Phys. Rev. B 46, 10903 (1992); Sh. Farhangfar, R.S. Poikolainen, J.P. Pekola, D.S. Golubev, and A.D. Zaikin, Phys. Rev. B 63, 075309 (2001).

18 G. Schön and A.D. Zaikin, Phys. Rep. 198, 237 (1990).

19 D.S. Golubev and A.D. Zaikin, Phys. Rev B 59, 9195 (1999).

20 M. Büttiker, Phys. Rev. B 46, 12485 (1992).

21 A.D. Zaikin, Physica B 203, 255 (1994).

${ }^{22}$ L.S. Levitov, H. Lee, and G.B. Lesovik, J. Math. Phys. 37, 4845 (1996). 